\title{
INTERPRETIERTES CHRISTENTUM? KRITISCHE RELEKTÜRE VON RATZINGERS EINFÜHRUNG IN DAS CHRISTENTUM
}

L UCIE K O L ÁŘ OVÁ

\section{ABSTRACT \\ Christianity Interpreted? A critical re-reading of Ratzinger's Introduction to Christianity}

Ratzinger's early work Introduction to Christianity presents an attempt at rational understanding of what theology considers to be crucial for Christianity and what should be thought over in the context of the (then) new challenges. The open character of the book can be understood in light of the fact that anthropological discourse was already well established in post-Council theology. For Ratzinger, this seems to be connected with a necessity to outline clear contours for Christianity. The book is one of the author's significant works with a wide impact, and which also represents Ratzinger's way of thinking in its relation to philosophical thought.

The title of this contribution is also its point: Ratzinger's book offers a certain interpretation of Christianity and provides at the same time a sort of defence of Christianity against a never-ending chain of interpretations. It is just that which should be subjected to interpretation.

\section{Keywords:}

Christianity; Theological anthropology; Credo; Philosophy; Interpretation

DOI: $10.14712 / 23363398.2020 .63$

\section{Ratzingers Buch Einführung in das Christentum wurde} in seiner Zeit (Erstausgabe in 1968) zum theologischen Bestseller. Etwas später hat es mit Küngs Christ sein (1974) und Rahners Grundkurs des Glaubens (1976) um die Gunst der Leser gewetteifert. Es wurden viele Exemplare verkauft, das Buch erwarteten wiederholte 
Veröffentlichungen. Schon nach acht Jahren war es in zwölf Sprachen übersetzt. ${ }^{1}$ Noch in 2000, also mehr als dreißig Jahre später, stimmte Joseph Ratzinger, damals Kardinal, einer unveränderten Veröffentlichung zu. Das erregt Aufmerksamkeit.

Mit einem inmitten Studentenmeutereien der sechziger Jahre und unmittelbar nach dem Zweiten Vatikanischen Konzil geschaffenen Buch, von dem man gerecht vermuten kann, es zeige das „geöffnetere“ Antlitz seines Autors, identifiziert sich der "spätere Ratzinger" ohne zu zögern. Also derjenige, der inzwischen rückhaltlos skeptisch der Methode nie zu endenden Interpretationen des christlichen Glaubens gegenübersteht und für den jeder weitere Stoß von Aggiornamento vor allem mit der Bedrohung verbunden ist, die Wahrheit relativiere sich. Im einleitenden Essay zur Neuausgabe bekennt R. sich zur Grundorientierung des Werks und setzt dieses frühe Bündel gar in die Kontinuität seines Denkens ein, ${ }^{2}$ also auch der Bücher, die eine Anklage gegen die moderne Theologie mitklingen lassen. ${ }^{3}$ Einführung kann man so zu Recht für „ratzingerisch wesenseigen“ halten: es repräsentiert nämlich sein theologisches Denken eines bestimmten philosophischen Zuschnitts und widerspiegelt seine theologische Tiefeneinstellung. Von der er selbst nachgerade sagt, die habe sich nie wirklich geändert. ${ }^{4}$

Den anklagenden Ton hört man gleich im Vorwort zur ersten Auflage mit. Die Ausgangsfrage nach Inhalt und Sinn des christlichen Glaubens in unsicheren Zeiten wird hier direkt von Befürchtungen vor Ausverkauf des Christentums begleitet. Attraktiv versteckt hinter dem Volksmärchen „Hans im Glück“, dabei aber deutlich anwesend als Befürchtung davor, das Christentum werde sich unter Wert verkaufen, werde leer. ${ }^{5}$ In weiteren Kapiteln drängt sich dem Leser die aufgeworfene Befürchtung auf und profiliert sich gleichsam zum epistemologischen Faden, sozusagen

1 Vgl. Hansjürgen Verweyen, Joseph Ratzinger - Benedikt XVI. Die Entwicklung seines Denkens (Darmstadt: Wissenschaftliche Buchgesellschaft, 2007), 43.

2 Vgl. Joseph Ratzinger, „Einführung in das Christentum - gestern, heute, morgen“. Vorwort zur Neuausgabe 2000, Einführung in das Christentum (München: Kösel, 2007), 9-26.

3 Z.B. Unterwegs zu Jesus Christus (Augsburg: Sankt Ulrich Verlag, 2003); Der Gott Jesu Christi. Betrachtungen über den Dreieinigen Gott, (München: Kösel, 2006).

4 Vgl. Joseph Ratzinger, Zur Lage des Glaubens, ein Gespräch mit Vittorio Messori (München: Neue Stadt, 1986), 16. Auch z.B. S. Wiedenhofer konstatiert keinen prinzipiellen Unterschied zwischen R.s früherer und späterer Theologie - weder im Inhalt noch in der Form. Vgl. Siegfried Wiedenhofer, Die Theologie Joseph Ratzingers/Benedikts XVI. Ein Blick auf das Ganze (Regensburg: Friedrich Pustet, 2016), 21, 27, 91-92, 136.

5 Vgl. Joseph Ratzinger, Einführung in das Christentum (München: Kösel, 1968), 9. 
zum Schattenmotiv des ganzen Buches. Gerade dieses Bewusstsein ermöglicht bei kritischer Lesung eine tiefere Orientierung in dem scheinbar zugänglichen und gut verständlichen Text, der doch ein Labyrinth bleibt - weil im Hintergrund von bestimmten axiomatischen, nicht immer offen konzeptualisierten Voraussetzungen geführt, die als solche kaum zu Dialog gestellt werden, wonach das Buch - vorgelegt als eine Art Aufforderung - sonst strebt. ${ }^{6}$

Wieso ist dann redlich zu vermuten, Einführung erschließe jenes geöffnete Antlitz seines Autors? Anscheinend ist es R. gelungen die gerade zu der Zeit reifen Fragen anzurühren. Einen der Zugangsschlüssel zur Verständigung bietet die hermeneutische Deutung, die es als Zeugnis von der katholischen Theologie der sechziger Jahre erfasst. Diese Theologie hat schon, mehr oder weniger, die anthropologische Wende durchgemacht ${ }^{7}$ - so, wie sie sie in ihren wiederherstellenden Strömungen seit den ersten Jahrzehnten des 20. Jahrhunderts ansteuerte und wie diese dann durch das Zweite Vatikanische Konzil besiegelt wurde. ${ }^{8}$ Die methodologische Nutzung dieses Faktes - da die Wende in der Theologiegeschichte zweifellos zu einer greifbaren Tatsache wurde - ermöglicht uns ein Instrument zu gewinnen, um R.s Buch rückwirkend in einen anthropologischen Kontext zu setzen und es in ihm neu zu lesen. Denn gerade auch dieses Buch ist ein Beleg dafür, dass die gegebene Wende im Rahmen des theologischen Denkens tatsächlich eintraf, dieses Denken verwandelte und unter anderem verursachte, dass theologische Antworten im öffentlichen Raum neu resonierten.

Im ersten Schritt werde ich daher - ohne Vollständigkeit zu beanspruchen - analysieren, worin der anthropologische Ansatz des Textes liegt. Der zweite Schritt soll die zugrundeliegende Struktur des Buches überprüfen und im dritten Schritt untersuche ich die philosophischen Wurzeln des jeweiligen theologischen Entwurfs. Somit glaube ich einen kritischen Zugang zur Lektüre von R.s Buch vorlegen zu dürfen. Der Text konzentriert sich fast ausschließlich auf das verfolgte Buch und bringt somit eine nur verknappte Sicht, die um einen breiteren Kontext zu ergänzen wäre. Zum Stand der Kirche und Theologie heuzutage kann nur sporadisch hingewiesen werden.

\footnotetext{
6 Vgl. Ratzinger, Einführung; z.B. 64-66.

7 Vgl. Karl Rahner, Schriften zur Theologie VIII. (Einsideln: Benzinger Verlag, 1967), 43.

8 Vgl. namentlich die pastorale Konstitution Gaudium et spes.
} 


\section{Die anthropologische Art des Buches}

R.s Ausgangspunkt ist Subjekt in seiner Einstellung zu Gott, Subjekt unter der Bekehrungsanforderung. Einem Supranaturalismus gegenüber, wo die Offenbarungsinhalte dem Menschen angebracht werden, thematisiert R. den Glauben primär als eine freie personale Tiefenentscheidung. Sein Ansatz zum religiösen Glauben ist existenziell. ${ }^{9}$

Verglichen mit der vorkonziliaren Dogmatik, die stark ecclesiozentrisch orientiert war, argumentiert R. verschiedentlich fundamental anthropologisch:

wenn er zwischen „Religion“ und „Glauben“ unterscheidet: zwischen Religion als einer Lebensordnung bzw. einem System von bestimmten rituellen Formen, Gepflogenheiten und deren Einhalten einerseits, und zwischen Glauben als einer Haltung, die nicht der Relation Wissen/ Machen, sondern der von Stehen/Verstehen entspricht anderseits; ${ }^{10}$

wenn er zwischen „Vernunft“ und „Glauben“ unterscheidet: zwischen Wissen, dessen Evidenz durch Beschränkung auf das Erscheinende, Sichtbare und somit die Wirklichkeit Verschmälernde erkauft ist, und Glauben, der gerade nicht auf der Ebene der Erkenntnis, sondern der tiefst menschlichen Existenzialität vortritt; ${ }^{11}$ so dass dann behauptet werden kann: „Jeder Mensch muß in irgendeiner Form zum Bereich der Grundentscheidungen Stellung beziehen, und kein Mensch kann das anders als in der Weise eines Glaubens tun. Es gibt einen Bezirk, der keine andere Antwort als die eines Glaubens zulässt, und gerade ihn kann kein Mensch ganz umgehen. Jeder Mensch muß auf irgendeine Art 'glauben'."12;

oder wenn er betont, das Christentum sei - anderen möglichen Entscheidungsformen des menschlichen Geistes gegenüber - ,vor allem auch eine Option für den Menschen“"13, inklusive seiner Transzendenz. Diese andere, von R. nur angedeutete Formen meinen die von der Menschheit immer wieder bereitgestellten Deutungsmuster (wie etwa kosmisches Chaos, Schicksalhaftigkeit, Determinismus, Nihilismus, kollektives Wohlergehen, Reinheit einer bestimmten Gemeinschaft u.a.), die dem Menschen als freiem Wesen mit unbedingter Würdigkeit nicht Genüge tun.

\footnotetext{
Vgl. Ratzinger, Einführung, 27-28.

Vgl. Ratzinger, Einführung, 25-26n., 43f.

Vgl. Ratzinger, Einführung, 33-52.

Ratzinger, Einführung, 46.

Ratzinger, Einführung, 121.
} 
Die anthropologische Argumentation ist nachspürbar im der Trinität gewidmeten Kapitel. Den Ausgangspunkt der Problematik legt R. ins Gebiet der Erfahrungen, mitnichten des Spekulativen. Erfahrungen bestimmter Art, die Menschen im Zusammenhang mit Glauben erlebten, streben an einem historischen Augenblick danach, auf zugehörige theologische Weise abgehandelt zu werden. ${ }^{14}$

Deutlich ist die anthropologische Verankerung in den christologischen Teilen des Buches, wo nicht irgendwelches, vom gläubigen Christen anzunehmendes System zum Ausgangspunkt wird, sondern die Person Jesu Christi. In ihr ist Existenz identisch mit Sendung, Botschaft, Wort. Der statischen und einbahnigen Inkarnationschristologie gegenüber ist für R. der Kreuz richtunggebend: Jesus opfert sich selbst, die Kreuzigung wird zu Herrschaft, Herrschaft beruht in der Kreuzigung. ${ }^{15}$ Der springende Punkt lässt sich hier vielleicht so formulieren: von Anthropologie ist nicht die Rede weil Gott Mensch geworden ist, sondern weil er als Mensch Gott war. Dasjenige, was auf der rein menschlichen Ebene zu Abgang würde, bekommt auf der Ebene der Beziehung zwischen Gott und Mensch sogar die entgegengesetzte Bedeutung.

Auch dort, wo R. die Stichhaltigkeit kirchlicher Struktur verteidigt, zeigt sich die anthropologische Lage als fundamental. Gott soll sicher nicht in Institutionen, Ereignissen, Worten, Riten verweilen. Er berührt zuallererst jeden Menschen in seinem Inneren und braucht im Prinzip keine vermittelnden Zwischenstufen. Dies ist dennoch nicht in subjektivistischer Verengung zu verstehen. R. eilt sich, zu unterstreichen, der wirkliche Mensch sei kein atomisiertes Individuum, er kommt gar nicht in den Blick als eine Einsamkeit des Ich. Zu sich selbst kommt der Mensch nur als leiblich beteiligt an dem Ganzen, als Mit-Sein. Natürlich ist Gott unabhängig von den äußeren Apparaturen, aber Christen nähern sich ihm geschichtlich in dem an, was 'Tradition' genannt wird. ${ }^{16}$ Es steckt eine Dialektik in R.s Argumentation: einerseits hört man im Appell an die Größe der Gemeinschaft eine radikale Orientierung auf den Einzelnen mitschwingen („Kirche und Christentum überhaupt sind um der Geschichte willen da, der kollektiven Verstrickungen wegen, die den Menschen prägen; sie sind

14 Vgl. Ratzinger, Einführung, 125f.

15 Vgl. Ratzinger, Einführung, 164-165.

16 Vgl. Ratzinger, Einführung, 198-207. 
auf dieser Ebene zu verstehen. Ihr Sinn ist es [...] das kollektive Gitter aufzubrechen oder zu verwandeln, das den Ort menschlicher Existenz bildet.“17), andererseits soll das Netz der menschlichen Gegenseitigkeit vor der Falle des Individualismus schützen („Weil das Christentum auf das Ganze bezogen ist und nur von der Gemeinschaft her und auf sie hin verstanden werden kann, weil es nicht Heil des isolierten einzelnen, sondern Indienstnahme für das Ganze ist, dem er weder entfliehen kann noch darf, eben darum kennt es in letzter Radikalität ein Prinzip 'einzelner'. “18) Die Pointe dieser Dialektik liegt ersichtlich darin, dass das Institutionelle, die Strukturen, wie solidarisch sie auch immer sein mögen, nie ohne den Einzelnen auskommen.

Allerdings stellt R. den anthropologischen Ansatz immer wieder unter das Primat der Gottesoffenbarung, unter das Geheimnis Gottes verborgener Anwesenheit. Die tiefste Schicht jedes Menschen stellt Gott dar - der führt ab von allerlei künstlichen Konstruktionen, Rekonstruktionen, Schöpfungen, Interpretationen, klugen Erklärungen, in denen der Mensch nur um das herumkreist, was in Wirklichkeit angenommen werden soll. ${ }^{19}$ Gott ist der Ausgangspunkt und der Höhepunkt des Menschen, Theologie ist die Letztbegründung der Anthropologie. In dieser Logik geht die anthropologische Perspektive nie in den Humanismus über, weil sie in keinem Moment aufhört, eine theologische Perspektive zu sein. Nicht überraschend im Gewirbel der sechziger Jahre, vielleicht aber überfokussiert, bricht R. der Theologie durchgehend ihre potential anthropozentrische Spitze ab, damit die Sache des Menschen nicht ihre eigenen Limiten überwuchern würde. Der dazu gebrauchte Hinweis zu Gottesoffenbarung dient R. trotzdem nicht als Alibi für die Entmachtung des Menschen, wie weiter ersichtlich wird.

Die anthropologische Sichtweise tritt im Text ebenfalls dort ein, wo zwischenkonfessionelle Themen berührt werden. Sie wird zum Hauptnenner für Größen, die zu R.s Zeit als inkompatibel verstanden wurden. Im Moment, wo es im Bereich der Ökumene so gut wie keinen Fortschritt gab - und dazu im Kontext, in dem Konfessionalität, Erbe der Neuzeit und Moderne, die grundlegende theologische Perspektive (und natürlich auch Kontroverse) war - überschreitet R. die konfessionelle Optik und spricht explizit von „Christentum“, nicht „Katholizismus“.

17 Ratzinger, Einführung, 202.

18 Ratzinger, Einführung, 204.

19 Vgl. Ratzinger, Einführung, 137-139. 
Er selbst reagiert eher schon auf eine andere, mehr aktuelle Kontroverse der späten Moderne, nämlich auf die Frage des Glaubens bzw. Unglaubens. Diese geht er existenziell an: „Niemand kann dem andern Gott und sein Reich auf den Tisch legen, auch der Glaubende sich selbst nicht. Aber wie sehr sich auch der Unglaube dadurch gerechtfertigt fühlen mag, es bleibt ihm die Unheimlichkeit des 'Vielleicht ist es doch wahr'. [...] Der Glaubende wie der Ungläubige haben, jeder auf seine Weise, am Zweifel und am Glauben Anteil [...]. Keiner kann dem Zweifel ganz, keiner dem Glauben ganz entrinnen [...]. ${ }^{620}$ Die Frage nach der konfessionellen Zugehörigkeit verschärft R. als Frage nach dem Menschen: „Nicht der konfessionelle Parteigenosse ist der wahre Christ, sondern derjenige, der durch sein Christsein wahrhaft menschlich geworden ist. Nicht derjenige, der ein Normsystem sklavisch und einzig auf sich selbst gedacht einhält, sondern derjenige, der frei geworden ist zur einfachen, menschlichen Güte.“21

Der anthropologische Ansatz des Buches wird so unter anderem durch Orientierung an Subjekt und Personalität, existenziellen Zugang, Erfahrungsbasis, Überkonfessionalität oder Rücksichtnahme auf den zeitgenössischen und aktuellen Kontext und die damit verbundene Offenheit für gelebte Dimension der Realität gekennzeichnet. ${ }^{22}$ Nur dass es mit Kennzeichen schwierig ist. Sie können nicht materialisiert werden, das Verfahren ist nicht schematisch. Zum Gegenstand weiterer Untersuchung soll die mehr oder weniger versteckte Epistemo-logik R.s Textes sein.

\section{Zur Schattenstruktur}

Die sichtbare Buchstruktur folgt dem Inhalt vom Apostolischen Glaubensbekenntnis als regula fidei nach. Parallel lässt sich eine subtile Linie erkennen.

Die jeweilige Wahl erklärt R. dadurch, dass das Credo sehr früh im Zusammenhang mit Taufspendung beheimatet wurde und man an ihm den anfänglichen Sinn von Lehre, Bekenntnis und Dogma sieht. ${ }^{23}$

20 Ratzinger, Einführung, 23-24.

21 Ratzinger, Einführung, 221.

22 Zur Charakteristik R.s Theologie insgesamt vgl. den kurzen Überblick von Josef Kreiml, ,,Mitarbeieter der Wahrheit.' Theologische Grundüberzeugungen des Papstes Benedikt XVI., " in Christliche Antworten auf die Fragen der Gegenwart. Grundlinien der Theologie Papst Benedikts VXI., ed. Josef Kreiml (Regensburg: Pustet, 2010), 9-34.

23 Vgl. Ratzinger, Einführung, 58-59. 
Es ging um einen in Rom verwendeten Text, dessen endgültige Form jedoch nicht im Laufe des zweiten oder dritten, sondern definitiv erst neunten Jahrhunderts fixiert wurde, wenn Karl der Große ihn zur Festigung seines Reiches als vereinigenden Faktor nutzte. H. Verweyen erinnert daran, dass die Angelegenheit also nicht frei von politischen Konnotationen ist, wie übrigens aus der darauffolgenden Entwicklung zur Uniformität der „römischen“ Kirche abzulesen ist. ${ }^{24}$

Das Credo selbst wird von R. nicht reduktiv dargestellt - etwa als ein ausschließlich verbindlicher, lehramtlicher Text. Er bringt ihn komplex dar: ein Symbol, das zwar auch die Lehre oder das später genannte Dogma einschließe, primär aber als Symbol des Glaubens da ist. Es enthalte die ganze anthropologische Breite des Glaubens. In ihm gehe es um die Antwort des Glaubens auf den zu Aufforderung gewordenen Jesus. In dem Symbol erklinge der ursprüngliche Dialog zwischen Gott und Menschen und zwischen Menschen untereinander - im Rahmen des Taufgeschehens. In ihm verberge sich das Wort als gehört, gesprochen, geschrieben, in ihm verberge sich Bekenntnis, es sei der Grund der Einheit, die es bewirke. ${ }^{25}$ Die politische Dimension der Sache wird nicht in Kauf genommen. Das Credo repräsentiere den christlichen Glauben nicht als politisches Manöver, nicht als philosophische Idee, sondern als Leben.

Wer aufmerksam liest, registriert, dass innerhalb einzelner Kapitel Gedanken ausgespielt werden, die mit der vorgelegten und als solcher gut zugänglichen Struktur nicht direkt zusammenhängen, oder wo der Zusammenhang gerade vorausgesetzt wird, ohne angedeutet, geschweige thematisiert zu sein. Dem dadurch beunruhigten Leser bleibt es dies aufzudecken. In einer in der Theologischen Revue (1969) veröffentlichten Rezension schrieb W. Kasper lapidar: „Die Darstellung ist eher meditativ und intuitiv als argumentierend und reflektierend. R. umkreist sein Thema, spielt mit den verschiedenen Motiven und führt sie dann meist überraschend zur Synthese [...]. Auf diese Weise entwirft er ein eindrucksstarkes Bild, aber die harten Konturen des logischen Gedankengangs sind nicht immer leicht herauszufinden. Der Leser erhält relativ wenig Information über den Stand der Diskussion, über Gründe und Gegengründe, sowie objektive Beurteilungskriterien. Dem theologisch nicht informierten ist es deshalb wohl nicht immer

${ }^{24} \mathrm{Vgl}$. Verweyen, Joseph Ratzinger - Benedikt XVI., 45.

25 Vgl. Ratzinger, Einführung, 54-69. 
deutlich, was sichere These und was bloße Hypothese, was gemeinsame kirchliche und theologische Lehre und was persönliche Theologie des Verfassers ist. Dadurch ist er der oft recht persönlich geprägten Sicht des Autors weitgehend ausgeliefert, zumal andere Meinungen oft von vorneherein in recht gezielter Akzentuierung zu Wort kommen. “26

Findet man in der Einführung eine Erklärung, um das Gesagte abzuschwächen? Im Vorwort spricht R. zwar von den den Charakter des Buches prägenden Umständen, die aber nur die äußeren sind: der Text ist aus den Vorlesungen für Tübinger Studenten, Hörer aller Fakultäten, im Sommersemester 1967 hervorgegangen, wissenschaftliche Belege wurden nur insoweit angefügt, als sie unmittelbar zur Vorbereitung des Verfassers dienten. ${ }^{27}$ Angesichts der oben angeführten Kritik bleibt der Eindruck etwas verlegen. Ja, die Verweise auf Literatur sind eher enthaltsam, nicht selten vermittelt, ${ }^{28}$ offenbar geht es insgesamt um eigene Besinnungen des Autors, sicherlich gebaut auf den von ihm schon früher interiorisierten Kenntnissen theologischer, philosophischer und historischer Art. Der wirkliche Stolperstein, den Kaspers Kritik trifft, liegt voraussichtlich woanders. Auf der ersten Ebene spricht R. offen und zugänglich, der Unterton ist jedoch konfrontativ. Als ob er sich von jemandem und etwas abgrenzen, gegen etwas kämpfen würde. Dargebrachte Verknüpfungen sind stellenweise mit dem Credoinhalt verbunden, oft wurzeln sie aber im Motivationsboden ihres Verfassers. ${ }^{29}$ Gerade dann identifiziert man sie als Antworten auf unausgesprochene

26 Walter Kasper, „Das Wesen des Christlichen,“ Theologische Revue 65, 3 (1969): 182188 , hier 184.

27 Vgl. Ratzinger, Einführung, 10.

28 Z.B. auf M. Luther wird via P. Hacker hingewiesen (168, Anm. 12); Aristoteles wird via H. Meyer zitiert (208, Anm. 43); einige Seiten werden dem Beitrag des Teilhard de Chardins gewidmet, aber die Fußnoten weisen auf C. Tresmontant hin und seine Einleitung in das Denken des jeweiligen Theologen (191-194 und Anm. 26-33). Der präsentierte de Chardin ist ziemlich verwässert, es geht um R.s Interpretation von Tremontants Interpretation, jedoch mit dem Anspruch, es handle sich um eine unmittelbare Paraphrase. Es wird auch nicht kritisch reflektiert, dass sowohl bei de Chardin (mit seinem unbestreitbaren Genie), als auch bei Tresmontant (in seiner Inklination zu ideologisierenden Deutungen) an die methodologischen und epistemologischen Grenzen gestoßen wird.

29 S. Wiedenhofer weist darauf hin, dass R. primär keine großen systematischen Entwürfe entwickelt, sondern es bei ihm wesentlich um eine situative und kontextuelle Theologie geht, um Gelegenheitsschriften, die auf konkrete Herausforderungen und Situationen reagieren. Im Rahmen des Handlungszusammenhangs ist R.s Theologie großenteils als „Krisenbewältigung“ und „Orientierungskontroverse“ zu verstehen. Vgl. Wiedenhofer, Die Theologie Joseph Ratzingers/Benedikts XVI., 23-24. 
Fragen, Rechtfertigungen gegen imaginäre Kläger, Ausfallschritte auf Gegner. Ich weise auf einiges hin.

\subsection{Die Figur des Dilemmatischen}

Im ersten Kapitel, auf dem Hintergrund des Dilemmas „traditionelle Theologie versus moderne Welt“, eröffnet R. die Metapher von einem Clown-Theologen, der - anstatt Theologie zugänglicher zu machen mittels seiner Gewänder die Aufmerksamkeit anderer zu sekundären Wahrnehmungen ablenkt. ${ }^{30}$ Es wird richtig gezeigt, dass es letztendlich nicht um Formverwandlung gehen kann, die durch irgendeinen visuellen Trick Theologie modernisieren würde. Es wird jedoch nicht ausgearbeitet, worin die echte Verwandlung wohl liegen könnte, bzw. sollte, ohne im gegebenen Dilemma stecken zu bleiben. Der Autor thematisiert nur, worin die echte und tiefe Theologie liegt, nämlich im „Scandalum“. Falls es weiterhin um das Christliche gehen soll, so R.s Annahme, darf keine theologische Verkleidung das primäre Ärgernis beseitigen. Theologie kann sich nie wirklich anpassen und es entsteht die Frage, ob ein zeitgemäßes Design überhaupt nachzustreben ist, oder ob vielleicht schon die Bestrebung um das Aggiornamento als solche die Entleerung der Theologie mit sich bringe... Aus zugespitzten Aussagen („Wenn etwa irgendwo ein Theologe erklärt, ,Auferstehung der Toten' bedeute nur, dass man täglich unverdrossen von neuem ans Werk der Zukunft zu gehen habe, so ist der Anstoß sicherlich beseitigt.“"31) wird der Leser nicht erfahren, wie man das Aggiornamento wohl machen kann, ohne sich am Christlichen zu veruntreuen. R.s Gedankenablauf lässt allmählich einsehen, dass gerade wegen der - unveränderlichen - Substanz des Christentums nicht einmal die Aufhebung des einschlägigen Dilemmas wünschenswert ist. Als ob Theologie für die Welt inakzeptabel bleiben sollte.

Das aufgeworfene Dilemma ist für R. nicht bloß ein methodisches Hilfsmittel, sondern eine grundsätzliche Aussage. „In der Fremdheit des theologischen Unterfangens den Menschen unserer Zeit gegenüber wird der, der seine Sache ernst nimmt, [...] die bedrängende Macht des Unglaubens inmitten des eigenen Glaubenswollens erfahren und erkennen. “32 Auch wenn sowohl Gläubigen als Ungläubigen

30 Vgl. Ratzinger, Einführung, 17-19.

31 Ratzinger, Einführung, 32.

32 Ratzinger, Einführung, 19. 
sich in nicht sehr unterschiedlicher Situation befinden, gibt es hier eine spezifische Fremdheit der Theologie, bzw. des Glaubens. Verursacht diese vielleicht, dass der Gläubige, sich des Ärgernisses des Christlichen bewusst, sich inmitten der „modernen Ungläubigen“ dementsprechend fremd fühlen muss? Aber was mit denen, die den Glauben ernst nehmen und sich fremd fühlen inmitten der „unmodernen Gläubigen"? Soll dies zu Beweis werden, dass sie die Sache nicht ernst genug nehmen? Ist das Dilemma, das die durch Ärgernis gezeichnete Theologie notwendigerweise mit sich bringt, gerade das tatsächlich Maßgebende für die Sache Christi? Könnte es nicht eher so sein, dass der ernst erlebte Glaube den Menschen gerade zum Leben zwischen anderen Menschen befähigt - und also auch zwischen seinen Zeitgenossen, die die altmodische Theologie nicht verstehen?

Das attraktiv angespielte Thema des Glaubens in der gegenwärtigen Welt klingt bei R. halbwegs skeptisch, sogar abwertend. Seiner Furcht gegenüber, das Christentum sei billig verkauft, kann die Furcht vor einem kompromisslosen, entmenschlichten Christentum gestellt werden. Die abschreckende Möglichkeit eines Christentums ohne Religion erwähnt R. sattsam. Aus der Geschichte kennt man aber auch die umgekehrt abschreckende Möglichkeit des „Übermaßes am Religiösen“, angebracht oder nicht. Das Risiko einer oberflächlichen Weitergabe berechtigt als solches die Instandhaltung des Status quo nicht. Die Eigenschaften des Tiefen/Oberflächlichen, des Authentischen/Unglaubwürdigen, des Wahrhaftigen/Falschen folgen keiner Dichotomie zwischen früher/jetzt oder dem Traditionellen/Modernen ${ }^{33}$, sie überqueren sie. Im Prinzip findet bzw. vermisst man das Ärgernis sowohl bei Theologie in den längst aus der Mode gekommenen Kleidern, als auch in der Verkleidung eines Clowns, der zum Lachen bringt, auch wenn er es ernst meint. Gerade weil die Essenz woanders liegt, braucht man einem modernen oder alternativen Gewand keine magische Macht zuzusprechen, geschweige es zu fürchten.

\subsection{Die Figur des Oppositionellen}

Einem dualistischen Schema entsprechend taucht an vielen Stellen des Buches „ein Gegner" auf - oft in Gestalt eines Angehörigen der „weltlichen Welt“, öfters aber als ein Christ bestimmter Prägung. Den

33 Vgl. Ratzinger, Einführung, 29f. 
weltlichen Gegner betreffen verschiedene Randbemerkungen, die ihn als einen Fortschrittler („wie wenig das [die christliche Eschatologie] in Wahrheit mit fröhlicher Fortschrittsromantik zu tun hat" $)^{34}$ oder gar als verkörperten Marxist zeigen. ${ }^{35}$ Es kann auch um einen - einigermaßen schematisierten - Kritiker der Kirche gehen, unabhängig ob weltlich oder christlich geprägt: „Im Grunde ist immer ein versteckter Stolz wirksam, wo die Kritik an der Kirche jene gallige Bitterkeit annimmt, die heute schon anfängt, zum Jargon zu werden." Es wirkt verstimmend, dass solche Sätze einer Überlegung über der unheiligen Heiligkeit der Kirche folgen. Warum, fragt der Leser, an dieser Stelle wieder ein Geplänkel?! „Leider gesellt sich nur allzu oft eine spirituelle Leere dazu, in der das Eigentliche der Kirche überhaupt nicht mehr gesehen wird, [...]. Die wirklich Glaubenden messen dem Kampf um die Reorganisation kirchlicher Formen kein allzu großes Gewicht bei. Sie leben von dem, was die Kirche immer ist. ${ }^{\text {" }}{ }^{6}$ R. darf hier eine spirituelle, zumeist an die Kritik der Kirche gebundene Leere diagnostizieren. Insgesamt legt er Kritik der Kritik vor, die leider auch von der galligen Bitterkeit gekennzeichnet zu sein scheint. Dabei, nur einige Seiten vorher, hat er gleichsam revolutionär getönt: „Das Zweite Vatikanische Konzil selbst hat sich dazu durchgerungen, nicht mehr bloß von der heiligen, sondern von der sündigen Kirche zu sprechen; wenn man ihm dabei etwas vorwarf, so höchstens dies, daß es noch viel zu zaghaft darin geblieben sei.“"37 Die „Dogmatik der Sündigkeit“ ist, R. zufolge, vor allem deswegen sehr gut nachvollziehbar, weil sie mit der Erfahrung jedes von uns übereinstimmt. ${ }^{38}$ Warum vermag dann derselbe R. die Kritik in die eigenen Reihen nicht zu ertragen, die er darüber hinaus selbst pauschal in den Mund der hypothetischen Kritiker hineinlegt?

Der Gegner aus den christlichen Kreisen begehe am häufigsten die Flucht in die Historie, zum historischen Jesus, reduziert auf bloß menschliches Phänomen. Anstatt in den seit Jahrhunderten gültigen Glauben zu vertrauen, fange dieser nachträglich an zu rekonstruieren, was nicht rekonstruierbar sei - da es nämlich nicht um Lehre oder Taten gehe, sondern letztendlich um das Annehmen der Person Jesu

\footnotetext{
Ratzinger, Einführung, 196.

Vgl. Ratzinger, Einführung, z.B. 218-219.

Ratzinger, Einführung, 286.

Ratzinger, Einführung, 282.

38 Vgl. Ratzinger, Einführung, 282-287.
} 
als Christi. ${ }^{39}$ Es ist fraglich, inwieweit R. die historische Frage nach dem irdischen Jesu für implizites Teil der Theologie hält. Dass „hohe Christologie“ ohne die reale historische Person Jesu von Nazareth zur Ideologie werden könnte, befürchtet er nicht so sehr. ${ }^{40}$ Dafür äußert er die Angst, die historische und phänomenologische Methode bedecke die Tiefe des Menschen und Gottes, und die „Exaktheit“ - ähnlich wie bei der naturwissenschaftlichen Methode - werde mit Wahrheitsverlust bezahlt. ${ }^{41}$ Gilt es aber nicht, die verschiedenen Ansätze in ihren unterschiedlichen Methoden und den damit verbundenen Ansprüchen zu respektieren? Möglicherweise begeht R. hier den Fehlgriff, aus einer methodologischer Einstellung der Historie („ohne Wahrheit“) auf eine prinzipielle Folgerung („Wahrheit gibt es nicht") ihrerseits zu schließen. Doch, wenn „Wahrheit“ im methodologischen Rahmen eines Ansatzes nicht als eine Kategorie da ist, kann sie hier auch nicht verlorengehen. ${ }^{42}$

\subsection{Die Figur der Konfrontation}

R. zählt sich selbst wohl zu den Theologen, die die Resultate der historisch-kritischen Exegese für die systematische Theologie für brauchbar halten. Darin unterscheidet er sich von denen, die ohne sie auskommen - wie Barth, von Balthasar oder auch Rahner. ${ }^{43}$ Wenn er aber die Frage nach dem historischen Jesus als modernes Klischee paraphrasiert, tut er es spöttisch und mit Bissigkeit: „Halten wir uns nicht dabei auf, dass eine so inhaltslose Botschaft, mit der man vorgibt, Jesus besser zu verstehen, als er sich selbst verstand, schwerlich jemandem

39 Vgl. Ratzinger, Einführung, 158-164.

40 Zu diesem Problem siehe Edward Schillebeeckx, Mensen als verhaal van God (Baarn: H. Nelissen, 1989), 123-124.

41 Vgl. Ratzinger, Einführung, 154-156.

42 R. Deines macht darauf aufmerksam, R. verteidige als sachlich angemessen eine Diktion, wo es zwischen historischer Rekonstruktion und theologischer Interpretation nicht sauber getrennt und unterschieden wird. So seien die Heilstatsachen historisch. Vgl. Roland Deines, „Der ,historische“ ist der ,wirkliche‘ Jesus. Die Herausforderung der Bibelwissenschaften durch Papst Benedikt XVI. und die dadurch hervorgerufenen Reaktionen,“ in „Mitarbeiter der Wahrheit.“ Christuszeugnis und Relativismuskritik bei Joseph Ratzinger/Benedikt XVI. aus evangelischer Sicht, ed. Christoph Raedel (Göttingen: Brunnen, 2013), 20-66, hier 37-42. In seiner Untersuchung der Rolle des Historischen in R.s Werk (vor allem das Jesus-Buch des Papstes) bringt Deines die Problematik nuanziert dar und weist andererseits auch auf das Berechtigte und Auffordernde in R.s Vorschlag des „schlichten“ Zugangs hin, der den christlichen Wahrheitsanspruch im historischen - fleischgewordenen Gottessohn - Jesus zu bewähren sucht. Vgl. ders., 59-63.

43 Vgl. Verweyen, Joseph Ratzinger - Benedikt XVI., 51-52. 
etwas hätte bedeuten können. Hören wir lieber einfach zu, wie es weitergegangen sein soll. Aus Gründen, die nicht mehr recht zu rekonstruieren seien, sei Jesus hingerichtet worden und als ein Scheiternder gestorben. Danach sei auf eine auch nicht mehr recht erkennbare Weise der Auferstehungsglaube entstanden [...] Alsbald - so sieht es unser Klischee - sei aber dann die Botschaft aus der semitischen Welt in die hellenistische übergangen. [...] Im hellenistischen Bereich waren diese Kategorien unverständlich; man habe folglich dort zu hellenistischen Vorstellungsmodellen gegriffen. [...] Das Ganze ist für den historisch Denkenden ein absurdes Gemälde, [...], für meinen Teil gestehe ich [...] lieber und leichter zu glauben imstande bin, dass Gott Mensch wird, als dass ein solches Hypothesen-Konglomerat zutrifft.“44 R. unterstellt der Theologie, „das Christliche in wohlklingende Allgemeinheiten aufzulösen“" ${ }^{45}$. Ihm zufolge wurde Jesus ,gleichsam auf das Gespenst des bloßen Dass abgemagerten historischen Jesus" ${ }^{* 46}$ verringert und gerade das sollte gezeigt haben, „, dass uns der Rückweg zu einem bloßen Jesus unwiderruflich versperrt ist. [...] Die Jesus-Romantik ist deshalb im letzten [...] zukunftslos und gegenwartsleer. ${ }^{647}$ Der Streit zwischen Historikern scheint für R. verwirrt zu sein, jedenfalls will er „dem eilfertigen Subtraktionsverfahren“48 in der Jesusforschung mit gebührender Kritik gegenüberstehen. Man kann sich natürlich fragen, ob es überhaupt in den Kräften der liberalen „Jesuologie-Vertreter“ sein konnte, Jesus zum Philanthropen zu machen, der durch seine Versöhnlichkeit, auf Kosten der Wahrheit, Widersprüche ausgleiche. Setzen vielleicht die aus der Jesusforschung hervorgegangenen und benannten Aspekte die Botschaft Jesu herab? Kann die Missachtung wirklich dieser Ebene entspringen? Es gibt doch eine ganze Skala theologischer Interpretationen, die den Kern des Christlichen, das Scandalum, verschieden behandeln ohne es weg zu interpretieren.

Man kann mit R. restlos übereinstimmen, im Grunde gehe es immer um Sinn und Wahrheit ${ }^{49}$ - gerade diese Voraussetzung ermöglicht es übrigens von Vereitlung, Missbrauch oder Entleerung zu reden. Die Frage nach der Wahrheit scheint jedoch bei R. an Angst gekoppelt zu

\footnotetext{
Ratzinger, Einführung, 171-173.

Ratzinger, Einführung, 198.

Ratzinger, Einführung, 159.

Ratzinger, Einführung, 160.

Ratzinger, Einführung, 164.

Vgl. Ratzinger, Einführung, z.B. 33, 47, 50, 69, 299.
} 
sein. Seine späteren Werke bringen hervor, dass die in diesem Buch identifizierbaren Anläufe sich bei ihm allmählich zu Angst vor Relativierung der Wahrheit profiliert haben. Auch wenn die Risiken einer Relativierung deutlich sind und wenn aus der christlichen Sicht eine Pluralität, die nicht unterscheidet, Toleranz erfordert und diese vielleicht sogar mit Achtlosigkeit gleichstellt, unakzeptabel ist, steht das ganze Phänomen der theologischen Forschung und der damit verbundenen Pluralität noch nicht unter Verdacht, die Reinheit des Glaubens $\mathrm{zu}$ bedrohen, ${ }^{50}$ und soll daher auch nicht als ein relativistisches Unternehmen disqualifiziert werden. Wenn R. den Relativismus später als eine gemeinsame Diagnose nahezu aller Krankheiten von Welt, Kirche und Theologie stellt, ${ }^{51}$ ist es inzwischen immer deutlicher, dass hier faktisch nicht zwischen einem dogmatistischen und verantworteten Relativismus unterschieden wird. ${ }^{52}$ Die Zurückweisung einer verantworteten Relativierung kommt dann selbst dogmatisch vor.

Dies alles lässt ahnen, warum R. dem Konzept der Geschichtlichkeit skeptisch gegenüber steht, oder beziehungsweise Theologie als politisch negiert. ${ }^{53}$ Die Welt lasse sich nämlich weder als factum erklären, noch als faciendum schaffen. Allerlei Wissenschaften, von

50 „Ich bewundere da immer wieder die Gewandtheit von Theologen, denen es gelingt, genau das Gegenteil von dem zu vertreten, was in klaren Dokumenten des Lehramtes geschrieben steht, um dann doch diese Umkehrung mit geschickten dialektischen Kunstgriffen als die ,wahre' Bedeutung des betreffenden Dokumentes hinzustellen.“ Ratzinger, Zur Lage des Glaubens, 24.

51 Joseph Ratzinger, Glaube - Wahrheit - Toleranz. Das Christentum und die Weltreligionen (Freiburg: Herder 2004), 94-98f. Joseph Ratzinger, Werte in Zeiten des Umbruchs. Die Herausforderungen der Zukunft bestehen (Freiburg: Herder, 2005), 51f., 114.

52 Der unverantwortlichen Willkür gegenüber hält der verantwortete Relativismus oder Pluralismus am Wahrheitsanspruch fest, andere Ansätze werden aber nicht als bedrohlich wahrgenommen. Vgl. Konrad Hilpert, Zentrale Fragen christlicher Ethik (Regensburg: Friedrich Pustet, 2009), 240-242. Die jeweilige Unterscheidung macht z.B. auch K. Koch nicht, der - übereinstimmend mit R. - ausschließlich von „Diktatur des Relativismus“ spricht. Vgl. Kurt Koch, ,,Was ist Wahrheit?“ - Dogma des Relativismus oder Frage auf Leben und Tod? Versuch einer Replik,“ in „Mitarbeiter der Wahrheit," 221-237.

53 Aufs Ganze gesehen gehört Geschichtlichkeit schon zu einem bleibenden wichtigen Thema bei R., das freilich verschieden rezipiert wird. Es gebe gelegentlich nicht nur zu viel Dogmatisches im Historischen, wie im oben genannten Sinn (Anm. 42), sondern „Geschichte“ trete bei R. auch an die Stelle der Gnade, womit - auf Kosten des Übernatürlichen - eine ungeheuerliche Profanierung begehen werde. Vgl. Paul Hacker, „Joseph Ratzinger und die Zerstörung des Dogmas, “ in Zur Philosophie und Theologie Joseph Ratzingers, ed. Wigand Siebel (Saarbrücken: SAKA, 2005), 14-30, hier 16. 
der Tendenz zur Geschichte betroffen, kommen mit Rekonstruktionen an, die doppeldeutig und immer mit Zweifel verbunden sind. ${ }^{54}$ Die Schöpfungswirklichkeit erkenne der Mensch nur auf der Ebene bloßer Fakten und „[...] nur die Erde der Fakten bleibt in seinen Händen zurück “55. Auch die historisierende Theologie laufe diese Gefahr. Sie resultiert in eine Pluralität irgendwie mangelhafter Deutungen. Nicht einmal das folgende Programm wird geglaubt: „Das Factum hat das Faciendum, das Gemachte hat das Machbare und Wiederholbare, Nachprüfbare aus sich entlassen und ist nun um seinetwillen da. ${ }^{“ 56}$ Welt kann man nicht rekonstruieren, nicht schaffen. Den Glauben auf der Faciendum-Ebene zu verstehen heißt für R. die Versuchung, die Techne auch in die Theologie einzusetzen - und somit zu vergessen, dass der Mensch sich nie zu Gott machen kann. Als theologisch problematisch befürchtet R. jede Manipulation mit dem Menschen und weigert sich, Theologie als Mittel zur Verwandlung der Welt zu politisieren. $^{57}$

Geschichtlichkeit ist ihm eine Falle. Was wirklich bleibt, sei der Glaube. Glaube, der zu mir von außen kommt, den ich nicht restlos erklären und auf keine Weise schaffen kann, der - von seiner tiefsten Art her - ein Empfangen ist. Glaube als Angebot, ursprünglicher als Denken. ${ }^{58}$ Theologie kann nicht anders als den Glauben denken, bei R. auf kontroverse Weise.

\section{3. Über den philosophischen Hintergrund}

Innerhalb der Frage, welche Stelle in R.s theologischer Besinnung die Philosophie einnimmt, sollen es zwei Ebenen unterschieden werden: seine eigene philosophische Position und (s)eine Metareflexion bezüglich Philosophie in ihrem Bezug auf Theologie.

\subsection{Ratzingers philosophische Position}

R. selbst bekennt sich im Buch zu keinem konkreten philosophischen Hintergrund, so dass der Leser hier über keinen Vor-zugang

\footnotetext{
Vgl. Ratzinger, Einführung, 36-40.

Ratzinger, Einführung, 38.

Ratzinger, Einführung, 40.

Vgl. Ratzinger, Einführung, 39-41.

58 Vgl. Ratzinger, Einführung, z.B. 47-48, 61f., 217-220.
} 
verfügt; der Text jedoch scheint ausgeprägt platonisch zu sein. ${ }^{59}$ Das gilt nicht nur dort, wo explizit philosophische Fragen behandelt werden, sondern gerade auch für den theologischen Kontext. R. entdeckt in Platon ein prophetisches, bis zu Christus angreifendes Potenzial und vermutet, Platons ernst philosophisches Denken habe als ob von vornherein etwas von den Offenbarungswegen erahnt. ${ }^{60}$ Infolge jeweiliger Einstellung kommen im Buch häufig dualistische Kategorien vor: das Nichtzusehende/das Sichtbare, Geistliches/Materielles, Geist oder Seele/Körper, ${ }^{61}$ die mehr als bloße Instrumente einer epistemologischen Unterscheidung seien: „Man begreift jetzt, wie der Glaube für ihn [R.] zu einem geradezu weltanschaulichen Bekenntnis zum Primat des Logos über die bloße Materie, des Geistes über den Bios zur gedanklichen, ja logischen Struktur des Seins, zur Welt als objektiver Geist, zur Antwort auf die Frage nach dem Ursinn von Einheit und Vielheit, von absolut und relativ [...] werden kann. “62

Es scheint problematisch zu sein, dass R. diese Kategorien als „gegeben“ positioniert, ohne die Berechtigkeit einer solchen Annahme zu erklären, geschweige zu widersprechen. „Der latente Idealismus und Säkularismus in R.s Einführung ist letztlich in seinem platonisierenden Ausgangspunkt begründet, durch den das eigentliche christliche Scandalum des, Logos sarx egeneto ${ }^{6}$ [Joh 1,14] gegen den immer wieder erklärten besseren Willen des Autors und trotz seiner ständigen Betonung der Positivität des Christlichen doch immer wieder unter die Vorherrschaft und unter die Gesetzlichkeiten des Wirklichkeitsbegriffs der griechischen Philosophie gerät. Die nachträgliche idealistische Synthese von Vernunft und Geschichte erlaubt es dann R. [...] das eine Mal existenzial, das andere Mal geschichtlich-leibhaftig zu interpretieren. Im gewissen Sinn wird er dadurch unangreifbar, weil er jedem Widerspruch gegen die eine Stelle eine andere entgegensetzen kann. Doch damit hebt sich die herausgestellte Positivität des Christlichen selber auf. R.s Intentionen widerstreiten der immanenten Logik seiner Kategorien und Argumente. Philosophische Grundsatzüberlegungen lassen sich in der Theologie nicht ungestraft übergehen. Wer meint, keine

\footnotetext{
${ }_{59} \mathrm{Zu}$ Grundlinien des philosophischen Denkens bei R. vgl. Ralph Weimann, Dogma und Fortschritt bei Joseph Ratzinger. Prinzipien der Kontinuität (Paderborn: Ferdinand Schöningh, 2012), 79-117.

60 Vgl. Ratzinger, Einführung, 240-242.

${ }^{61}$ Vgl. Ratzinger, Einführung, z.B. 27-28, 34, 48, 119-120, 190, 199-200, 208, $267 f$.

62 Kasper, Das Wesen des Christlichen, 185.
} 
Philosophie zu haben, bei dem wirken sich bestimmte Philosopheme um so unkontrollierter und verhängnisvoller aus.“63

Ein Indiz R.s philosophischer Präferenzen findet man in einer Fußnote, wo er zwischen den antiken Philosophen, die alle polytheistisch waren, Atheisten und Monotheisten unterscheidet, und sich zugleich darüber beschwert, dass dieser Tatbestand „unter der Herrschaft einer einseitig philosophiegeschichtlichen Betrachtungsweise selten genügend gewürdigt wird."64 Erst vor so einem Hintergrund sei nämlich das Revolutionäre der christlichen Haltung offensichtlich, da in ihr "die philosophische und religiöse Orientierung identisch werden. ${ }^{665}$ Methodologisch fragt das Letztgenannte jedoch nach Differenzierung: das metaphysische System, ein Konstrukt, ist nicht dasselbe wie Monotheismus; der allerletzte Grund des Seins ist nicht identisch mit dem einzigen Gott als Objekt der Ehrfurcht und des Glaubens. Die Frage nach dem Unterschied zwischen dem platonischen absoluten Sein (Begriff) und dem Gott des biblischen Glaubens stellt sich R. schon, ${ }^{66}$ die Antworten wirken dennoch pauschalisierend. Im Buch verstreut, basieren sie anscheinend auf einer vorausgesetzten Deutungsformel: die frühe Kirche hat eine historische Wahl im Rahmen der antiken Kultur gemacht, da sie sich für den Logos, gegen den Mythos, entschieden hat, und Gott, als das Sein selbst, mit dem philosophischen Grund alles Seins identifiziert hat. R. ist der Überzeugung, dass gerade die Ankoppelung vom Gott des Glaubens an den Gott der Philosophen eine glückliche war, infolgedessen die Welt und Religion definitiv entmythologisiert wurden. Und das Christentum wurde vor einem Zusammenbruch bewahrt. ${ }^{67}$ Nur dass in dieser Sichtweise die Selbsttragfähigkeit des auf der Person Jesu Christi gestellten Christentums geschwächt ist und die Ontologisierung der Theologie als beinahe unentbehrlich postuliert ist. ${ }^{68}$

Nur eine bestimmte Art Philosophie scheint so für R. relevant zu sein. In seinem Buch findet man keine Spur von kantischer Tradition, so maßgebend für die moderne Epoche, deren Erben R. anzusprechen

\footnotetext{
Kasper, Das Wesen des Christlichen, 185.

Ratzinger, Einführung, 78, Fußnote 7.

Vgl. Ratzinger, Einführung, 78, Fußnote 7.

Vgl. Ratzinger, Einführung, 96v.

Vgl. Ratzinger, Einführung, 103-105v.

Weiteres zum Verhältnis Glaube/Vernunft und zur Rolle der patristischen Theologie und Ontologie/Metaphysik in R.s Theologie vgl. Wiedenhofer, Die Theologie Joseph Ratzingers/Benedikts XVI., 242-273f, vor allem 258.
} 
versucht. Als ob für ihn die Genialität der griechischen Philosophie bzw. des sogenannten philosophischen Monotheismus einen Bogen nur noch ins Mittelalter zieht und nachher nur mehr in ausgewählten Traditionen durchscheint - in Hegels oder Kierkegaards Philosophie. Die Umgestaltung des philosophischen Ausgangspunkts seit der Neuzeit wird nicht thematisiert.

\subsection{Suchen nach der Verortung von Philosophie}

Wenn R. die Eigenart des Glaubens darstellt, tut er das unter anderem durch die Auseinandersetzung mit Philosophie. Während es im Glauben „einen Vorrang des Wortes vor dem Gedanken“ gibt, „geht in der Philosophie der Gedanke dem Wort voraus". ${ }^{69}$ Daran zeige sich die essenzielle Differenz zwischen ihnen: Philosophie geht aus der Initiative des Menschen hervor, so dass das von ihm ausgesprochene Wort das Resultat eines privaten Wahrheitssuchen - immer nur sekundär bleibt und als solches durch andere Wörter ersetzbar ist. Im Glauben kommt demgegenüber das Wort zum Menschen „von außen“, aus der Wirklichkeit. Solche Differenz macht einen wesentlichen Unterschied in Struktur aus: einmal individuell (der Einzelne sucht erst nachher Anhänger für seine Gedanken), einmal dialogisch (Gott ruft zuerst den Menschen).$^{70}$

Philosophie als Gedankenspiel kann nicht die Tiefe des Glaubens erreichen. Denn im Glauben geht es um innerliches Ringen, um Schutzschaltung gegenüber der Vergöttlichung des Weltlichen, um die Kraft, Leid zu ertragen. ${ }^{71}$ Für Philosophie ist Denken der absolute Wert, für Glauben steht die (absolute) Liebe am höchsten. Bezogen auf Wahrheit schreibt R. es folgendermaßen um: „Wenn Platonismus eine Idee von der Wahrheit gibt, so gibt christlicher Glaube die Wahrheit als Weg, und erst indem sie zum Weg wird, ist sie des Menschen Wahrheit geworden. Wahrheit als bloße Erkenntnis, als bloße Idee bleibt ohne Kraft; des Menschen Wahrheit wird sie erst als Weg, der ihn beansprucht, den er betreten kann und muß."72 Zur Verteidigung der Philosophie ist jedoch zu erwähnen, originär soll auch sie einen Weg (zur Weisheit) darstellen.

\footnotetext{
Ratzinger, Einführung, 62.

70 Vgl. Ratzinger, Einführung, 62-66.

71 Vgl. Ratzinger, Einführung, 79-83.

72 Ratzinger, Einführung, 69.
} 
Die Wendung „kann und muss betreten“ verweist weiter auf R.s Thema der Freiheit, die zum Unterscheidungsmerkmal des Christentums wird. Der christliche Glaube sei einmal eine Philosophie der Freiheit, einmal eine persönliche Wahl, nie eine (kosmisch-naturgesetzliche) Notwendigkeit. Genauer gesehen thematisiert R. die Freiheit weniger als eine subjektive Wahlmöglichkeit, dafür aber als Wahlmöglichkeit für etwas. Die letztere wird durch eine bestimmte objektive Dringlichkeit gekennzeichnet, denn das Leben in der Welt bringt schon von seiner „Konstruktion“ her diese Wahl notwendig mit sich. ${ }^{73}$ Jenseits strikter philosophischer Argumentation scheint in diesem Zusammenhang R.s kühner Vorschlag, es sei die Quadratur des Zirkels der Theologie realisierbar. Das Christentum müsse vom Menschen angenommen werden, das von außen zukommende Ereignis werde das dem Menschen Notwendige: „Das Inkognito Gottes als Mensch in der Geschichte 'muß' sein - mit der Notwendigkeit der Freiheit. "74 Appelliert R. hier an das Annehmen des christlichen Angebots als eine freie Entscheidung, oder geht es darum, sich dem zu unterwerfen, was unvermeidlich ist? Kasper zufolge erweist R. hier - zusammen mit Schelling und Hegel das geschichtlich Zufällige als das Notwendige ${ }^{75}$ Der Imperativ einer als notwendig thematisierten Freiheit scheint tatsächlich die Freiheit des Glaubens in das gerade Gegenteil umzukehren.

Durch dieses Paradox der Freiheit weist R. weiter darauf hin, dass Philosophie als solche durch den Glauben überschritten wird. Freiheit sei letztendlich nicht eine gedankliche Kategorie. Da die Welt keine Mathematik, sondern Liebe ist, kann das „mathematische Weltverständnis“ hier höchstens einen Gott der Philosophen finden. ${ }^{76}$ Das theologische Denken im Kontext des Glaubens ist - aufgrund der relationalen Natur Gottes - schöpferisch und sein absoluter Wert heißt soviel wie lieben. ${ }^{77}$ Durch die Wette auf die philosophische Ratio habe das Christentum eine epochale Entscheidung genommen und das philosophische Reflexionsniveau gehöre somit untrennbar zum christlichen Erbe. In ihrem autonomen Wert wird Philosophie desungeachtet von R. nur sparsam bedacht. Er setzt sich mit ihr verhältnismäßig dienstlich und markant selektiv auseinander. Verweyen zufolge ist dieser Status

\footnotetext{
Vgl. Ratzinger, Einführung, 121-123.

Ratzinger, Einführung, 220.

Vgl. Kasper. Das Wesen des Christlichen, 184.

7 Vgl. Ratzinger, Einführung, 116, 123.

7 Vgl. Ratzinger, Einführung, 112.
} 
quo in der späteren Entwicklung bei R. nicht überwunden, so dass er gerade auch einen polemisch kritischen Ton der Philosophie gegenüber angibt, mit der er sich vermutlich nicht einmal näher bekannt gemacht hat. ${ }^{78}$

\section{Schluß}

In Einführung bringt R. eine bestimmte Interpretation vom Christentum an. Gleichzeitig stellt das Buch auch den objektiven Inhalt des Christentums in seinem universalistischen Anspruch dar. Es scheint mir evident zu sein, dass die - deklarierte oder faktische - objektive Textebene tatsächlich noch nicht eine Interpretationsabwesenheit heißt, sosehr dies für R. im Widerspruch stehen möge. Im Buch spielt nämlich sein Begriff mit, der objektive Kern des Christentums wäre an sich verfügbar, als eine Art Harddisk, auf der normative Richtlinien abzulesen sind. R. selbst ist aber ein Beispiel davon, dass dieser objektive Kern in Wirklichkeit nie anders als durch eine Interpretation verfügbar ist.

R. würde sich lieber ohne das behelfen. Das Kriterium liege in Wahrheit, direkt zugänglich: „Aber was nur noch durch Interpretation bestehen kann, hat in Wirklichkeit aufgehört zu bestehen. Der menschliche Geist wendet sich mit Recht der Wahrheit selbst zu und nicht dem, was mit der Methode der Interpretation auf Umwegen als mit der Wahrheit noch vereinbar erklärt werden kann, selbst jedoch keine Wahrheit mehr hat.“ Die Vernunft hat zwar die Rationalität der Wahrheit zu verteidigen, um den Rückzug „in einen Bereich bloßer Frömmigkeit, bloßen Glaubens, bloßer Offenbarung“ zu vermeiden, gleichzeitig darf sie aber nicht von der Wahrheit abführen, in einem Verfahren, das R. „abkürzend als Interpretationschristentum bezeichnen möchte. Hier wird mit der Methode der Interpretation der Skandal des Christlichen aufgelöst und, indem es solchermaßen unanstöBig gemacht wird, zugleich auch seine Sache selbst zur verzichtbaren Phrase gemacht, zu einem Umweg, der nicht nötig ist, um das Einfache zu sagen, das hier durch komplizierte Auslegungskünste zu seinem Sinn erklärt wird“79.

${ }_{78}$ Vgl. Verweyen, Joseph Ratzinger - Benedikt XVI., 52.

79 Ratzinger, Einführung, 107. Es gilt für alle drei anschließende Zitate. 
Stimmt das theologisch? Eine Interpretation muss das christliche Ärgernis nicht unbedingt beseitigen, sie kann zu ihm auch - authentisch - herführen. Und andersrum. Das Scandalum des Christentums kann ebenfalls durch eine vermutliche Nicht-Interpretation verwischt werden, da die Übertragung des konservierten Kerns, den es in Wirklichkeit nie uninterpretiert gab ${ }^{80}$ an dem lebendigen Brennpunkt der Botschaft Jesu Christi auch total vorbeizugehen vermag. Konsequent durchdacht müsste man vor dem christlichen Kerygma schweigend stehen und ihm durch die Radikalität unserer Lebensgeschichten huldigen. Es wäre schwierig auch die Existenz der Kirche legitim zu verteidigen, die seit Anbeginn dieses Kerygma unter anderem dadurch bewahrt und weitergegeben hat, indem sie es interpretierte... Warum klassifiziert R. gleich im Vorwort als Gedankenlose jene, die „das Neue unbesehen jederzeit auch schon für das Bessere halten“81? Kann nicht auch das Alte vorgefasst für das Bessere gehalten werden?

Die Analyse zeigt, dass R. mit seiner Kritik des Interpretierens vor allem einen Anspruch bekämpft, den er dem interpretierenden Verfahren selbst zuerkennt: als ob eine Interpretation das Recht mit sich bringe, im Prozess des Interpretierens den Sinn dessen zu schaffen, was interpretiert wird. Während - so R. - der Sinn lediglich anzunehmen ist. Ist dieses Dilemma aber treffend? Der Sinn wird erst recht in einer Interpretation erschlossen. Es gehört zur Aufgabe der Interpretation, den Sinn zu entdecken und immer wieder zu aktualisieren, ja auch mit dem Risiko, es gelinge nicht besser. Das Christentum kann, soll, ja sogar muss sich selbst interpretieren, damit es immer wieder angeeignet werden könnte. Mit guten Interpretationen dient man hoffentlich der Sache Gottes, mit schlechten Interpretationen bringt man sie nicht zu Fall. Gerade ein solches basales Vertrauen ist es, was wohl im Buch mangelt.

Faculty of Theology University of South Bohemia in České Budéjovice

Kněžská 8

37001 České Budéjovice Czech Republic

E-mail: kolaroval@tf.jcu.cz

80 Vgl. Schillebeeckx, Mensen als verhaal van God, 54-63.

81 Ratzinger, Einführung, 9. 